\title{
Erratum to: Modeling of complex interfaces for pendant drop experiments
}

\author{
Caroline Balemans $^{1} \cdot$ Martien A. Hulsen $^{1}$ - Theo A. Tervoort ${ }^{2}$ - Patrick D. Anderson ${ }^{1}$
}

Published online: 19 May 2017

C Springer-Verlag Berlin Heidelberg 2017

\section{Erratum to: Rheol Acta (2016) 55:801-822 DOI 10.1007/s00397-016-0956-1}

The original version of this article unfortunately contained mistakes. Theo A. Tervoort was not listed among the authors. The correct information is given above.

In Balemans et al. (2016), an axisymmetric finite element model is presented to study the behaviour of complex interfaces in pendant drop experiments. While the bulk fluid of the pendant drop is modeled as a Newtonian fluid, the interface behaviour is described using a quasi-linear Kelvin-Voigt constitutive model. To study the influence of the five model parameters of this constitutive model, a parameter study is performed. Furthermore, small amplitude oscillatory measurements are simulated to demonstrate the applicability of the numerical model.

The quasi-linear two-dimensional Kelvin-Voigt viscoelastic constitutive equation as proposed by Verwijlen

The online version of the original article can be found at http://dx.doi.org/10.1007/s00397-016-0956-1.

Patrick D. Anderson

p.d.anderson@tue.nl

1 Polymer Technology, Eindhoven University of Technology, Eindhoven, The Netherlands

2 Institute für Polymere, ETH Zürich, Zürich, Switzerland et al. (2014) and used in Balemans et al. (2016) is not material frame indifferent, because the Cauchy-Green deformation tensor $\boldsymbol{C}_{\mathrm{s}}$ is directly coupled to the surface stress tensor $\boldsymbol{5}$. Instead the Finger tensor $\boldsymbol{B}_{\mathrm{s}}$ should be used to construct a material frame indifferent constitutive equation. The correct formulation of the quasi-linear twodimensional Kelvin-Voigt constitutive equation becomes

$$
\begin{aligned}
\boldsymbol{S}= & \gamma \boldsymbol{I}_{\mathrm{s}}+\kappa\left(\nabla_{\mathrm{s}} \cdot \boldsymbol{u}\right) \boldsymbol{I}_{\mathrm{s}}+\mu\left[\nabla_{\mathrm{s}} \boldsymbol{u}+\left(\nabla_{\mathrm{s}} \boldsymbol{u}\right)^{\mathrm{T}}-\left(\nabla_{\mathrm{s}} \cdot \boldsymbol{u}\right) \boldsymbol{I}_{\mathrm{s}}\right]+K \ln \left(J^{\frac{1}{2}}\right) \boldsymbol{I}_{\mathrm{s}} \\
& +G\left(J^{-\frac{1}{2}} \boldsymbol{B}_{\mathrm{s}}-\boldsymbol{I}_{\mathrm{s}}\right),
\end{aligned}
$$

where $J=\operatorname{det}\left(\boldsymbol{B}_{\mathrm{s}}\right)$. The constitutive equation has both viscous and elastic properties, and all simulations for Balemans et al. (2016) have been repeated after implementation of Eq. 1 into the numerical model. The results show minor differences for small deformations and small values of the surface elasticity parameters. However, the results presented in Figure 18 (only $K=100 \mu \mathrm{N} / \mathrm{mm}$ ) and Figure 21 are significantly different. These deviating results are summarized in Fig. 1, where the apex length in time for $G=1,10,50,100 \mu \mathrm{N} / \mathrm{mm}$ is shown at a surface tension $\gamma=50 \mu \mathrm{N} / \mathrm{mm}$, surface dilatational viscosity $\kappa=$ $1 \mu \mathrm{Ns} / \mathrm{mm}$, surface shear viscosity $\mu=1 \mu \mathrm{Ns} / \mathrm{mm}$, and surface dilatational elasticity $K=100 \mu \mathrm{N} / \mathrm{mm}$ as presented before using the Cauchy-Green tensor $\boldsymbol{C}_{\mathrm{s}}$ (dotted lines) and with the use of the Finger tensor $\boldsymbol{B}_{\mathrm{s}}$ (solid lines). Using the new results, Figures 18 and 21 of the article by Balemans et al. (2016) should be replaced with Figs. 2 and 3, respectively. The conclusions of the article do not change. 
Fig. 1 Apex length in time for different values of the surface shear elasticity $G$ at a surface dilatational elasticity

$K=100 \mu \mathrm{N} / \mathrm{mm}$, all other parameters are set according to the reference state. The dotted lines are for the erroneous formulation with $\boldsymbol{C}_{\mathrm{s}}$; the solid lines with Eq. 1

Fig. 2 (Revised Figure 18)

Apex length in time for different values of the surface dilatational elasticity $K$ using Eq. 1, all other parameters are set according to the reference state

Fig. 3 (Revised Figure 21)

Apex length in time for different values of the surface shear elasticity $G$ at a surface dilatational elasticity $K=100 \mu \mathrm{N} / \mathrm{mm}$ using Eq. 1, all other parameters are set according to the reference state
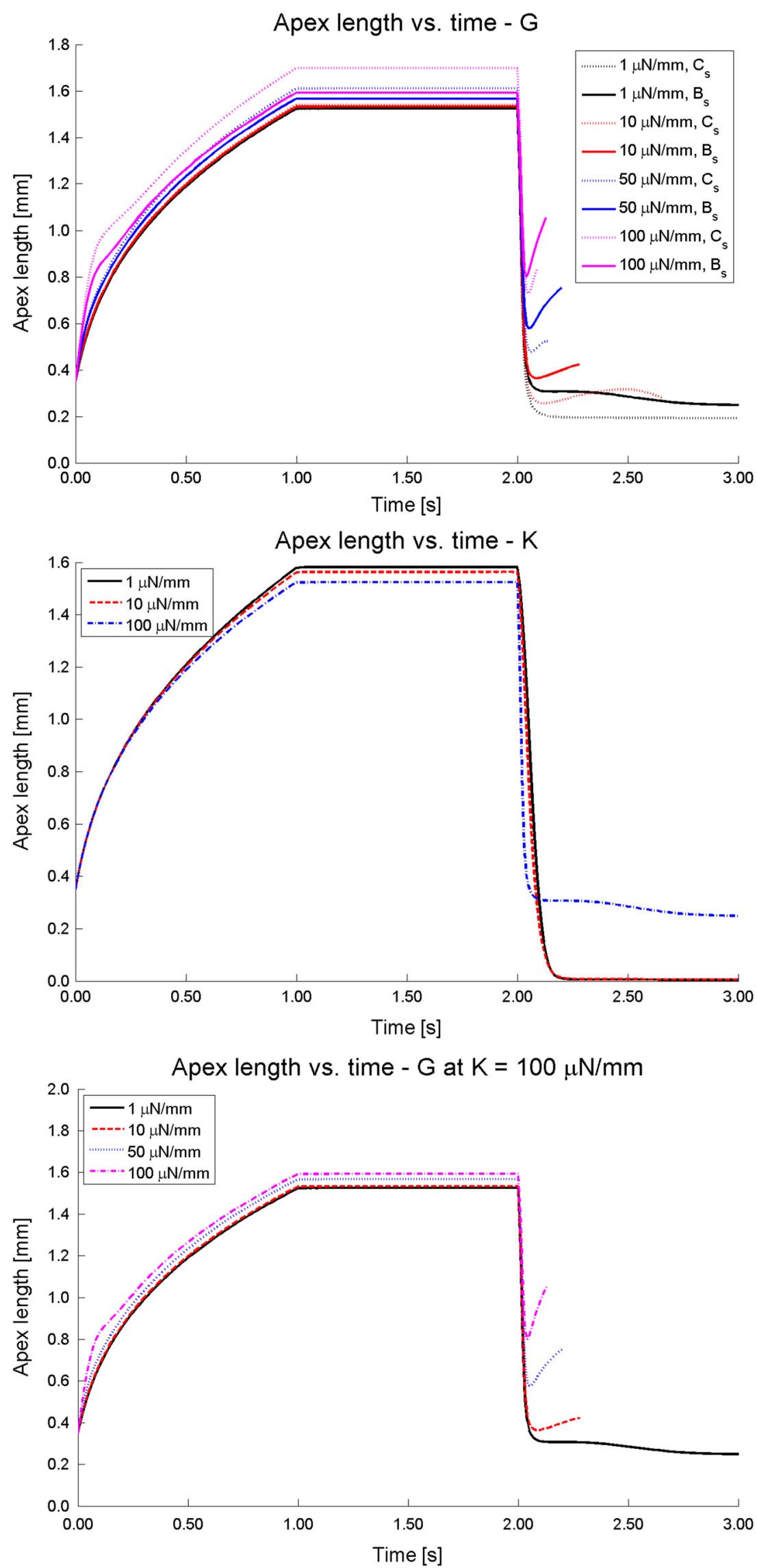


\section{References}

Balemans C, Hulsen M, Anderson P (2016) Modeling of complex interfaces for pendant drop experiments. Rheologica Acta 55:801822

Verwijlen T, Imperiali L, Vermant J (2014) Separating viscoelastic and compressibility contributions in pressure-area isotherm measurements. Adv Colloid Interface Sci 206:428-436 